\title{
Predicting loss of height in surgically treated displaced intra-articular fractures of the calcaneus
}

\author{
Manouk Backes $^{1}$ - Maarten C. Dorr ${ }^{1}$ - Jan S. K. Luitse ${ }^{1}$. \\ Johan C. Goslings ${ }^{1} \cdot$ Tim Schepers ${ }^{1}$
}

Received: 9 July 2015 / Accepted: 17 August 2015 / Published online: 15 September 2015

(C) The Author(s) 2015. This article is published with open access at Springerlink.com

\begin{abstract}
Purpose The goal of calcaneal fracture surgery is to restore its anatomy and good foot function. However, loss of height of the subtalar joint can occur post-operatively, as expressed by a decrease in Böhler's angle (BA). The aim of this study was to identify potential factors associated with a post-operative decrease in BA.

Methods All consecutive adult patients treated with open reduction and internal fixation (ORIF) by an extended lateral approach (ELA) between 2000 and 2013 were retrospectively included. Primary outcome was the occurrence of a calcaneal collapse, defined as a postoperative decrease of $\geq 10^{\circ}$ in BA. The BA was measured pre-operatively, directly following surgery and at one year follow-up. Patient characteristics (body mass index, diabetes mellitus, smoking/alcohol/substance abuse, American Society of Anaesthesiologist classification), fracture classification and treatment characteristics: peroperative increase in BA and occurrence of post-operative wound infection (POWI) were collected.

Results A total of 262 patients with 276 calcaneal fractures were included. A calcaneal collapse occurred in 46 cases (17\%). The median preoperative BA, per-operative increase in BA and post-operative decrease in BA were, respectively, $2^{\circ}, 27^{\circ}$ and $4^{\circ}$. A calcaneal collapse was seen more often following a per-operative increase of $>25^{\circ}$ in BA, but no significant association was found ( $p=0.056)$. Uni- and multivariate analysis showed that patients with substance abuse and
\end{abstract}

Manouk Backes

m.backes@amc.nl

1 Academic Medical Center, Trauma Unit, Department of Surgery, G4, University of Amsterdam, Meibergdreef 9, PO Box 22660, 1100

DD Amsterdam, The Netherlands those with POWI had significantly more calcaneal collapse $(p<0.05)$. No association was found between substance abuse and the occurrence of POWI $(p=0.293)$.

Conclusions In nearly one in six patients with an intraarticular calcaneal fracture treated with ORIF by an ELA, a post-operative collapse of $\geq 10^{\circ}$ was found during follow-up. Calcaneal collapse was correlated with the occurrence of a POWI and substance abuse.

Keywords Calcaneal fracture · Surgery · Böhler's angle · Extended lateral approach $\cdot$ Postoperative wound infection . POWI

\section{Introduction}

In 1931, Böhler proposed that radiological measurements of the calcaneus could be useful to evaluate initial damage as well as reduction quality in posterior-facet fractures of the calcaneus [4]. The Böhler's angle (BA) is the angle formed by the intersection of an imaginary line joining the highest point of the anterior calcaneal process and the highest point of the posterior talocalcaneal joint with a line drawn from the most superior point of the posterior talocalcaneal joint to the highest point of the calcaneal tuberosity measured on a lateral foot radiograph (Fig. 1). A normal BA ranges between 25 and $40^{\circ}[4$, $7,29]$ and a BA of $\leq 20^{\circ}$ following trauma is highly accurate for determining the presence of a calcaneal fracture [9].

The goal of calcaneal fracture surgery is to restore the anatomy of the calcaneus with its articular surfaces and thereby maintain foot function. However, the subtalar joint frequently collapses (calcaneal collapse) post-operatively to some extent, which can be seen at radiological follow-up [11, 14, 24]. Several authors noted a correlation between initial BA and outcome $[3,10,15,18,22,24]$. The BA at time of admission 

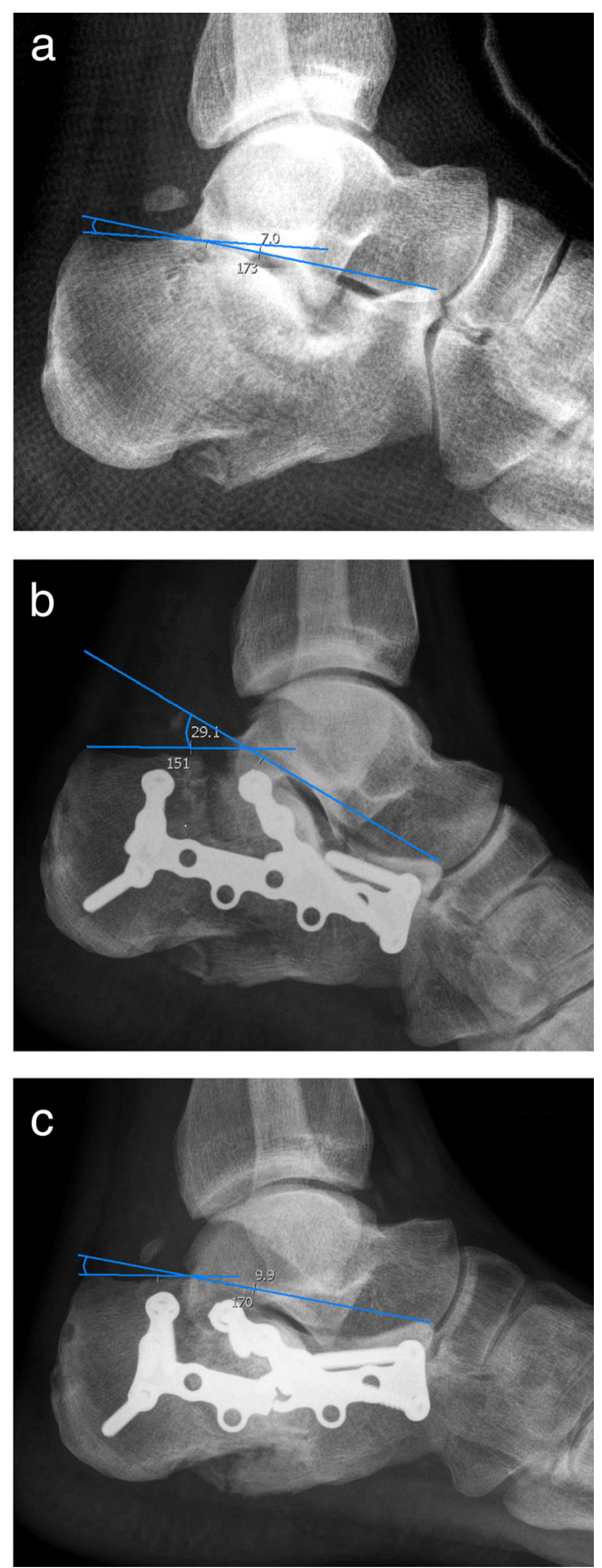

Fig. 1 Böhler's angle (BA) is the angle formed by the intersection of an imaginary line joining the highest point of the anterior calcaneal process and the highest point of the posterior talocalcaneal joint, with a line drawn from the most superior point of the posterior talocalcaneal joint to the highest point of the calcaneal tuberosity, as measured on lateral foot radiograph. a Preoperative BA of $7^{\circ}$. b Post-operative BA of $29^{\circ}$. c Calcaneal collapse of $10^{\circ}$ at one year follow-up

appears to be a valuable prognosticator for functional longterm results [18]. A poor clinical outcome is more common when the BA is decreased [10]. Surgical restoration of the BA leads to a better outcome compared with non-operative treatment $[15,17]$; however, operative overcorrection of a reduced BA should be avoided [18]. Patients treated conservatively have a larger definitive reduction in BA than patients treated operatively [3].

The aim of this study was to investigate the trend in Böhler's angle in patients with a displaced intra-articular calcaneal fracture treated surgically with an extended lateral approach (ELA) and to identify factors associated with a postoperative calcaneal collapse.

\section{Materials and methods}

The hospital database of a level 1 trauma centre was used to identify patients with calcaneal fracture surgery using the appropriate procedure code. All consecutive adult patients treated with open reduction and internal fixation (ORIF) via an ELA between 2000 and 2013 were retrospectively included. Exclusion criteria were patients with an open fracture who were initially treated with external fixation prior to ORIF, surgery by a different approach than an ELA, patients with a primary subtalar arthrodesis, referred patients with a preexisting wound infection and patients with reconstructive surgery following conservative treatment of a calcaneal fracture. Patients in whom a secondary arthrodesis was performed during follow-up were not excluded.

Primary outcome was calcaneal collapse, which was defined as a post-operative decrease of $\geq 10^{\circ}$ in BA. This cutoff value was chosen following an extensive literature search: in patients treated with ORIF, the highest reported decrease in BA ranged from 6 to $10.4^{\circ}$ at 1 -year follow-up $[6,11,14]$ and in patients treated conservatively an $11^{\circ}$ collapse was detected [3].

\section{Radiographic evaluation}

The BA was measured on a lateral radiograph, as described previously, at three different time points: pre-operatively, directly following surgery and at one year follow-up [22]. In patients in whom a secondary arthrodesis was deemed necessary, BA was measured prior to this procedure. All measurements were rounded to a full degree because of interobserver measurement reliability $[20,26,29]$ and were made by an independent observer. A specialised trauma surgeon verified all measurements. In case of discrepancies, measurements were averaged.

\section{Surgical procedure}

The surgical procedure was performed via an ELA, in which the full-thickness flap was retracted according to the no-touch technique, with temporary $\mathrm{K}$ wires in the talus to facilitate operative exposure [34]. A nonlocking stainless steel AO/ synthes calcaneal plate with $3.5-\mathrm{mm}$ stainless steel screws (Synthes, West Chester, PA, USA) was used [2, 21, 34]. The goal of surgery was restoration of articular surfaces, calcaneal height, width, length and correction of axis. No bone grafting 
or locking plates were used. Patients received a single administration of antibiotic prophylaxis preoperatively and thrombosis prophylaxis during their 12 weeks of nonweight bearing. No postoperative casting was used, and patients were instructed to perform flexion and extension exercises of the ankle [27].

\section{Clinical data}

Data were obtained from the electronic and paper medical records. The Institutional Review Board approved the study. Patient characteristics collected were gender, age at time of operation, body mass index (BMI), presence of diabetes mellitus (DM), smoking habits, alcohol use, substance abuse and American Anaesthesiologist Association (ASA) classification. Fracture-related characteristics were pre-operative $\mathrm{BA}$, which was subdivided in three groups $\left(<0^{\circ}, 0-15^{\circ}\right.$ and $\left.>15^{\circ}\right)$ [5] and fracture classification (Essex-Lopresti and Sanders). Treatment characteristics were per-operative increase in BA (post-operative $\mathrm{BA}$ minus pre-operative BA, subdivided into groups of $<10^{\circ}$, $10-25^{\circ}$ and $>25^{\circ}$ ), post-operative decrease in BA and occurrence of post-operative wound infection (POWI), subdivided into superficial and deep according to criteria of the United States Centers for Disease Control and Prevention $[2,16]$.

\section{Statistical analyses}

Data were analysed using the Statistical Package for the Social Sciences (SPSS) version 19.0 (SPSS INC., Chicago, IL, USA). Descriptive analysis was performed to assess baseline characteristics. For continuous data, medians with interquartile ranges (IQR) of p75-p25 or Q3-Q1 in nonparametric data were calculated; the Mann-Whitney $U$ test was used for analysis. Categorical data were compared using the chi-square test, and a $p$ value $<0.05$ was set as statistical significance. Firstly, a univariate analysis was performed, followed by a multivariate logistic regression analysis to model the relationship between different covariates and calcaneal collapse. Covariates with a $p$ value $<0.05$ were selected for multivariate logistic regression analysis.

\section{Results}

A total of 262 patients with 276 calcaneal fractures over the 14 -year study period were assessed. A post-operative calcaneal collapse of $\geq 10^{\circ}$ occurred in 46 cases $(17 \%)$. Median preoperative $\mathrm{BA}$ was $2^{\circ}(\mathrm{IQR}=13-10)$ (Fig. 2$): 42 \%$ of patients had a BA of $<0^{\circ}$ after trauma, followed by $38 \%$ with an initial BA between 0 and $15^{\circ}$, and $20 \%$ with a BA of $>15^{\circ}$. Median per-operative increase in $\mathrm{BA}$ was $27^{\circ}(\mathrm{IQR}=38-15)$ and median post-operative decrease in $\mathrm{BA}$ in 1 year was $4^{\circ}(\mathrm{IQR}=-2$ to -7$) ; 38 \%$ of patients had a post-operative decrease in BA of $>5^{\circ}$ and $6 \%$ of $>15^{\circ}$. Calcaneal collapse was seen more often

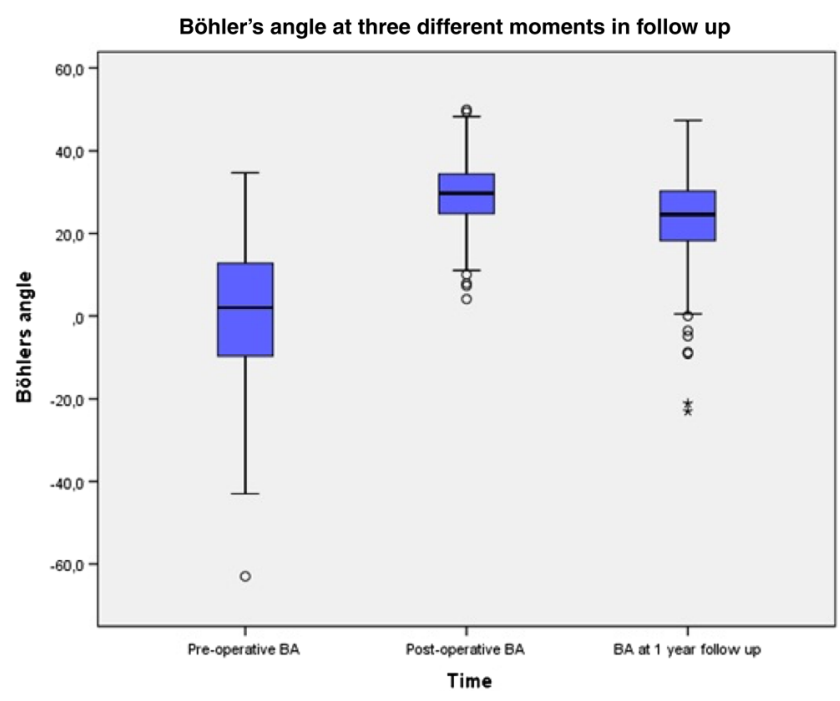

Fig. 2 Böhler's angle (BA) at three time points: median pre-operative, post-operative and at one year follow-up

following an increase of $>25^{\circ}$ in BA, per-operatively but no significant association was found $(p=0.056)$. Patient, fracture and treatment characteristics are shown in Table 1.

Univariate analysis showed that patients who suffered from a POWI $(n=70, ; 25.4 \%)$ and deep POWI $(n=34 ; 12.3 \%)$ had a calcaneal collapse more frequently (respectively, $p<0.001$ and $p=0.005$ ). These findings remained following multivariate logistic regression analysis (respectively, $p=0.035$ and $0.004)$. Also, patients with substance abuse $(n=55)$ had a calcaneal collapse significantly more frequent $(p=0.041)$. No association was found between substance abuse and the occurrence of POWI $(p=0.293)$.

\section{Discussion}

In nearly one in six surgically treated calcaneal fractures, calcaneal collapse $\geq 10^{\circ}$ occurred within one year, which was associated with POWI and substance abuse. Importantly, no association was found between substance abuse and the occurrence of POWI. The reason calcaneal collapse was found frequently in this cohort (17\%) at one year follow-up could be because no locking plates or bone-void fillers were used during surgery. This is a limitation of our study. Locking plates may provide better stability and functional recovery $[6,8]$ and less decrease of BA post-operatively [15]. Autologous bone grafting may aid in achieving and maintaining restoration of calcaneal height and anatomic reduction [1]; however, no objective radiographic or functional benefits of the use of bonegraft supplementation in the operative treatment of intraarticular calcaneal fractures was found [14]. A study by Johal et al. supports the use of an injectable in situ hardening calcium phosphate paste to fill the bone void after a displaced 
Table 1 Patient, fracture and treatment characteristics and incidence of calcaneal collapse in patients with calcaneal surgery

\begin{tabular}{lclll}
\hline & Patients $n(\%)$ & Collapse (\%) & No collapse (\%) & $P$ value (2-sided)** \\
\hline Number (\%) & 276 & $46(17)$ & $230(83)$ & NA \\
Male & $192(71)$ & $33(72)$ & $159(69)$ & 0.861 \\
Age at time of surgery (years) & $46(35-55)$ & $43(34-54)$ & $46(35-56)$ & 0.732 \\
Diabetes mellitus & $14(5)$ & $3(2)$ & $11(5)$ & 0.710 \\
BMI* & $24(22-27)$ & $24(23-27)$ & $24(21-27)$ & 0.168 \\
ASA 1 & $184(67)$ & $26(58)$ & $158(69)$ & 0.165 \\
Smoking & $108(41)$ & $17(40)$ & $91(41)$ & 1.000 \\
Alcohol use & $145(56)$ & $24(59)$ & $121(56)$ & 0.864 \\
Substance abuse & $55(22)$ & $15(35)$ & $40(20)$ & 0.041 \\
Fracture characteristics & & & & \\
Essex-Lopresti (TT) & $125(48)$ & $16(35)$ & $109(50)$ & 0.073 \\
Sanders (types 1 and 2) & $205(78)$ & $37(80)$ & $168(77)$ & 0.700 \\
Preoperative Böhler's angle & & & & \\
$<0^{\circ}$ & $93(42)$ & $16(40)$ & $77(42)$ & 0.861 \\
$0-15^{\circ}$ & $86(38)$ & $19(48)$ & $67(36)$ & 0.212 \\
$>15^{\circ}$ & $45(20)$ & $5(13)$ & $40(184)$ & 0.275 \\
Treatment characteristics & & & & \\
Surgical increase in Böhler's angle & & & & \\
$<10^{\circ}$ & $29(13)$ & $4(10)$ & $25(14)$ & 0.795 \\
$10-25^{\circ}$ & $99(44)$ & $14(35)$ & $85(46)$ & 0.222 \\
$>25^{\circ}$ & $126(56)$ & $28(70)$ & $98(53)$ & 0.056 \\
$\quad$ POWI & $70(26)$ & $24(52)$ & $47(21)$ & $<0.001$ \\
Deep POWI & $34(12)$ & $12(26)$ & $22(10)$ & 0.005 \\
\hline
\end{tabular}

$B M I$ body mass index, $A S A$ American Association of Anaesthesiologists, $N A$ not applicable, $N$ number, $P O W I$ postoperative wound infection, $T T$ tongue type

*Median with interquartile ranges

** Chi-square or Mann-Whitney $U$ test in patients with and without postoperative collapse intra-articular calcaneal fracture, as it shows less reduction in BA during follow-up [11].

It remains unclear how POWI is correlated with a postoperative decrease in BA. High rates of POWI were found, but numbers are in concordance with the literature on POWI following ORIF using the ELA [2, 32]. The occurrence of POWI could be the result of decreased vascularisation caused by the ELA and a calcaneal collapse due to subsequent delayed union. Shuler et al. found that patients with wound complications had a greater post-operative BA and a greater peroperative increase in BA than patients without wound complications. This is likely a result of increased tension on the wound edges [19, 23]. However, previous research by the investigators showed no correlation with the pre-operative BA or per-operative increase in BA and POWI [2]. In our study, calcaneal collapse occurred no more frequently if BA increased $>25^{\circ}$ during surgery, but no statistical significance was reached $(p=0.056)$. Our standard recommendation is 12 weeks of nonweight bearing following ORIF by the ELA until fracture healing is seen on radiographs or, when in doubt, on a computed tomography (CT) scan. As calcaneal collapse is associated with the occurrence of POWI, our data might indicate that prolonged immobilisation of these patients could be taken into consideration.

Importantly, in this study, we did not compare different surgical approaches. In current literature, a lower POWI rate of $6-14 \%$ is reported following the sinus tarsi approach [12, $30,31,33]$. This could be accompanied by a lower incidence of post-operative calcaneal collapse.

A calcaneal collapse in patients with substance abuse could be the result of limited compliance post-operatively, e.g. noncompliant weight bearing. However, as this was a retrospective study, we have no data to support this idea. Methods to increase fracture stability, such as primary arthrodesis, locking plates or bone-substitute materials, could be beneficial in patients in whom minimal compliance can be expected.

A general limitation of BA measurement is the risk of measurement errors due to interobserver variability or inability to correctly align the foot in standard radiographs; this misalignment can lead to errors in measurements [25, 28]. Interobserver variability in BA was classified as moderate [26] or good regarding agreement between independent observers [29]. 
Therefore, all radiographs were evaluated by two independent observers.

Radiographs of the contralateral calcaneus were not analysed because pre-operative templating of the uninjured contralateral calcaneus does not allow for more anatomic reduction or restoration of pre-injury morphology of the calcaneus undergoing operative fixation based on BA and calcaneal length [13].

Finally, we focused on radiographic analysis. Functional outcome, as measured using patient-reported outcome measures, was not used. Previous research showed that measurements on plain radiographs were not useful in determining outcome after intra-articular calcaneal fractures [22]. However, they are useful for determining fracture healing, alignment and surgical restoration of anatomy.

In conclusion, in nearly one in six patients with an intraarticular calcaneal fracture treated with ORIF through an ELA, a post-operative calcaneal collapse of $\geq 10^{\circ}$ was found during follow-up. Collapse was correlated with the occurrence of POWI and substance abuse. A post-operative CT scan may be advisable following 12 weeks of nonweight bearing to evaluate bone healing prior to weight bearing in this group of patients.

Disclosure No benefits in any form have been received or will be received from a commercial party related directly or indirectly to the subject of this article.

Open Access This article is distributed under the terms of the Creative Commons Attribution 4.0 International License (http://creativecommons.org/licenses/by/4.0/), which permits unrestricted use, distribution, and reproduction in any medium, provided you give appropriate credit to the original author(s) and the source, provide a link to the Creative Commons license, and indicate if changes were made.

\section{References}

1. Agrawal DP, Pawar ED, Lokhande V, Goyal S (2014) Bohler's angle: Correlation with outcome in displaced intra-articular calcaneal fractures treated with locking compression plate fixation with and without bone grafting 3:10946-10953. doi: 10:14260/jemds/ 2014/3429

2. Backes M, Schepers T, Beerekamp MS, Luitse JS, Goslings JC, Schep NW (2013) Wound infections following open reduction and internal fixation of calcaneal fractures with an extended lateral approach. Int Orthop. doi:10.1007/s00264013-2181-1

3. Bakker B, Halm JA, Van Lieshout EM, Schepers T (2012) The fate of bohler's angle in conservatively-treated displaced intra-articular calcaneal fractures. Int Orthop 36:2495-2499. doi:10.1007/s00264012-1706-3

4. Böhler L (1931) Diagnosis, pathology and treatment of fractures of the os calcis. J Bone Joint Surg Am 13:75-89

5. Buckley R, Tough S, McCormack R, Pate G, Leighton R, Petrie D, Galpin R (2002) Operative compared with nonoperative treatment of displaced intra-articular calcaneal fractures: a prospective, randomized, controlled multicenter trial. J Bone Joint Surg Am 84-A:1733-1744

6. Chen K, Zhang H, Wang G, Cheng Y, Qian Z, Yang H (2014) Comparison of nonlocking plates and locking plates for intraarticular calcaneal fracture. Foot Ankle Int 35:1298-1302. doi:10.1177/1071100714547520

7. Chen MY, Bohrer SP, Kelley TF (1991) Boehler's angle: a reappraisal. Ann Emerg Med 20:122-124

8. Hyer CF, Atway S, Berlet GC, Lee TH (2010) Early weight bearing of calcaneal fractures fixated with locked plates: a radiographic review. Foot Ankle Spec 3:320-323. doi:10.1177/1938640010374121

9. Isaacs JD, Baba M, Huang P, Symes M, Guzman M, Nandapalan H, Moopanar T, Marchalleck S, Szomor Z (2013) The diagnostic accuracy of bohler's angle in fractures of the calcaneus. J Emerg Med 45:879-884. doi:10.1016/j.jemermed.2013.04.055

10. Janzen DL, Connell DG, Munk PL, Buckley RE, Meek RN, Schechter MT (1992) Intraarticular fractures of the calcaneus: value of CT findings in determining prognosis. AJR Am J Roentgenol 158:1271-1274. doi:10.2214/ajr.158.6.1590122

11. Johal HS, Buckley RE, Le IL, Leighton RK (2009) A prospective randomized controlled trial of a bioresorbable calcium phosphate paste (alpha-BSM) in treatment of displaced intra-articular calcaneal fractures. J Trauma 67:875-882. doi:10.1097/TA. 0b013e3181ae2d50

12. Kikuchi C, Charlton TP, Thordarson DB (2013) Limited sinus tarsi approach for intra-articular calcaneus fractures. Foot Ankle Int 34: 1689-1694. doi:10.1177/1071100713510267

13. Kwon JY, Zurakowski D, Ellington JK (2015) Influence of contralateral radiographs on accuracy of anatomic reduction in surgically treated calcaneus fractures. Foot Ankle Int 36:75-82. doi:10.1177/ 1071100714552483

14. Longino D, Buckley RE (2001) Bone graft in the operative treatment of displaced intraarticular calcaneal fractures: is it helpful? J Orthop Trauma 15:280-286

15. Loucks C, Buckley R (1999) Bohler's angle: correlation with outcome in displaced intra-articular calcaneal fractures. J Orthop Trauma 13:554-558

16. Mangram AJ, Horan TC, Pearson ML, Silver LC, Jarvis WR (1999) Guideline for prevention of surgical site infection, 1999. hospital infection control practices advisory committee. Infect Control Hosp Epidemiol 20:250-78. doi:10.1086/501620, quiz 279-80

17. Paley D, Hall H (1993) Intra-articular fractures of the calcaneus. A critical analysis of results and prognostic factors. J Bone Joint Surg Am 75:342-354

18. Persson J, Peters S, Haddadin S, O'Loughlin PF, Krettek C, Gaulke $R$ (2014) The prognostic value of radiologic parameters for longterm outcome assessment after an isolated unilateral calcaneus fracture. Technol Health Care. doi: R2253462N8X0TG62

19. Sangeorzan BJ, Benirschke SK, Carr JB (1995) Surgical management of fractures of the os calcis. Instr Course Lect 44:359-370

20. Sayed-Noor AS, Agren PH, Wretenberg P (2011) Interobserver reliability and intraobserver reproducibility of three radiological classification systems for intra-articular calcaneal fractures. Foot Ankle Int 32:861-866

21. Schepers T, Den Hartog D, Vogels LM, Van Lieshout EM (2013) Extended lateral approach for intra-articular calcaneal fractures: an inverse relationship between surgeon experience and wound complications. J Foot Ankle Surg 52:167-171. doi:10.1053/j.jfas.2012. 11.009

22. Schepers T, Ginai AZ, Mulder PG, Patka P (2007) Radiographic evaluation of calcaneal fractures: to measure or not to measure. Skeletal Radiol 36:847-852. doi:10.1007/s00256-007-0330-6

23. Shuler FD, Conti SF, Gruen GS, Abidi NA (2001) Wound-healing risk factors after open reduction and internal fixation of calcaneal fractures: does correction of bohler's angle alter outcomes? Orthop Clin North Am 32:187-92 
24. Su Y, Chen W, Zhang T, Wu X, Wu Z, Zhang Y (2013) Bohler's angle's role in assessing the injury severity and functional outcome of internal fixation for displaced intra-articular calcaneal fractures: A retrospective study. BMC Surg 13:40-2482-13-40. doi: 10.1186/ 1471-2482-13-40

25. Touissant RJ, Gitajn L, Kwon J (2013) Measuring bohler's angle with oblique lateral radiographs: implications for management of calcaneal fractures. Harvard Orthopaedic J 15:7-12

26. Veltman ES, van den Bekerom MP, Doornberg JN, Verbeek DO, Rammelt S, Steller EP, Schepers T (2014) Three-dimensional computed tomography is not indicated for the classification and characterization of calcaneal fractures. Injury 45:1117-1120. doi:10.1016/ j.injury.2014.01.022

27. Wei SY, Okereke E, Esmail AN, Born CT, deLong WG (2001) Operatively treated calcaneal fractures: To mobilize or not to mobilize: $71-73$

28. Willauer P, Sangeorzan BJ, Whittaker EC, Shofer JB, Ledoux WR (2014) The sensitivity of standard radiographic foot measures to misalignment. Foot Ankle Int 35:1334-1340. doi:10.1177/ 1071100714549188

29. Willmott H, Stanton J, Southgate C (2012) Bohler's angle - what is normal in the uninjured british population? Foot Ankle Surg 18: 187-189. doi:10.1016/j.fas.2011.10.005
30. Xia S, Lu Y, Wang H, Wu Z, Wang Z (2014) Open reduction and internal fixation with conventional plate via L-shaped lateral approach versus internal fixation with percutaneous plate via a sinus tarsi approach for calcaneal fractures - a randomized controlled trial. Int J Surg 12:475-480. doi:10.1016/j.ijsu.2014.03.001

31. Zhang T, Su Y, Chen W, Zhang Q, Wu Z, Zhang Y (2014) Displaced intra-articular calcaneal fractures treated in a minimally invasive fashion: longitudinal approach versus sinus tarsi approach. J Bone Joint Surg Am 96:302-309. doi:10.2106/ JBJS.L.01215

32. Zhang W, Chen E, Xue D, Yin H, Pan Z (2015) Risk factors for wound complications of closed calcaneal fractures after surgery: a systematic review and meta-analysis. Scand J Trauma Resusc Emerg Med 23:18. doi:10.1186/s13049-015-0092-4

33. Zwipp H, Rammelt S, Amlang M, Pompach M, Durr C (2013) Operative treatment of displaced intra-articular calcaneal fractures. Oper Orthop Traumatol 25:554-568. doi:10.1007/ s00064-013-0246-3

34. Zwipp H, Rammelt S, Barthel S (2004) Calcaneal fractures-open reduction and internal fixation (ORIF). Injury 35(Suppl 2):SB4654. doi:10.1016/j.injury.2004.07.011 